\title{
Elección de carrera e institución de educación superior: validación de instrumento de medición mediante el modelado de ecuaciones estructurales
}

Choice of Career and Institution of Higher Education: Validation of the Measuring Instrument by Modeling Structural Equations

Escolha de carreira e instituição de ensino superior: validação de instrumento de medida por modelagem de equações estruturais

Rafael García Martínez

Tecnológico Nacional de México, Campus Guaymas, México

gamrmx@yahoo.com https://orcid.org/0000-0001-7175-5361

Eduardo Rafael Poblano-Ojinaga Tecnológico Nacional de México, Campus La Laguna, México pooe_65@hotmail.com https://orcid.org/0000-0003-3482-7252

Rigoberto Reyes Valenzuela Tecnológico Nacional de México, Campus Orizaba, México rigoreyes23@hotmail.com https://orcid.org/0000-0002-8570-8563

Guillermo Cuamea Cruz Universidad de Sonora, México guillermo.cuamea@unison.mx https://orcid.org/0000-0001-8884-6825 
Rubén Juárez Rodríguez

Tecnológico Nacional de México, Campus Orizaba, México rubenchojr@gmail.com https://orcid.org/0000-0003-0002-1787

\section{Resumen}

La elección de institución y carrera universitaria que realiza el egresado de la educación media superior es una variable que impacta en la deserción escolar, un indicador de calidad de las universidades. En la literatura revisada se proponen diferentes modelos que describen la relación de esta variable con un conjunto de factores, sin embargo, dichos modelos no son del tipo correlacional, por lo que no es posible establecer cuál de los factores tiene más peso o impacto ni la estructura de correspondencia entre ellos. En este trabajo se valida, mediante la técnica de los modelos de ecuaciones estructurales, un instrumento de medición que se utiliza para determinar si los datos recopilados son adecuados para verificar las hipótesis que indican la forma en que se relacionan las variables latentes o constructos con la variable "Elección de carrera" y "Universidad". Para validar este instrumento de medición se conformó una muestra de 157 alumnos que cursan la carrera de ingeniería Industrial de la Universidad de Sonora. En los resultados se descubrió que las variables latentes o constructos siguientes: Costos económicos, Servicios, Imagen de la universidad e Infraestructura son apropiados para modelar la variable "Elección de carrera" mediante un modelo de ecuaciones estructurales.

Palabras clave: análisis factorial confirmatorio, ecuaciones de modelos estructurales, elección de carrera universitaria, instrumento de medición, validez y confiabilidad del instrumento de medición. 


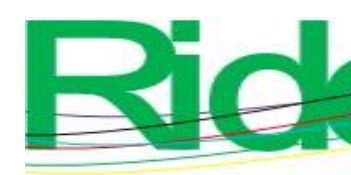

Revista Iberoamericana para la

Investigación y el Desarrollo Educativo

ISSN $2007-7467$

\section{Abstract}

The choice of institution and university career is a variable that impacts school dropout, an indicator of quality in universities. In the reviewed literature, different models are proposed that describe the relationship of this variable with a set of factors; however, these models are not of the correlational type, so it is not possible to establish which of the factors has more weight or impact or the correspondence structure between them. In this work, a measurement instrument that is used to determine whether the collected data is adequate to verify the hypotheses that indicate the way in which the latent variables or constructs are related to the variable "Choice of career" and "University". To validate this measurement instrument, a sample of 157 students who are studying Industrial Engineering at the Universidad de Sonora was formed. In the results, it was discovered that the following latent variables or constructs: Economic costs, Services, Image of the university and Infrastructure are appropriate to model the variable "Career choice" using a structural equation model.

Keywords: confirmatory factorial analysis, structural model equations, university career choice, measuring instrument, validity and reliability.

\section{Resumo}

A escolha da instituição e da carreira universitária feita pelo graduado do ensino médio é uma variável que impacta a evasão escolar, um indicador de qualidade nas universidades. $\mathrm{Na}$ literatura revisada, são propostos diferentes modelos que descrevem a relação dessa variável com um conjunto de fatores, porém esses modelos não são do tipo correlacional, não sendo possível estabelecer qual dos fatores tem mais peso ou impacto ou a estrutura de correspondência entre eles. Neste trabalho, um instrumento de medida que é utilizado para determinar se os dados coletados são adequados para verificar as hipóteses que indicam a forma como as variáveis ou construtos latentes se relacionam com as variáveis "Escolha de carreira" e "Universidade". Para validar esse instrumento de medida, foi formada uma amostra de 157 alunos que cursam Engenharia Industrial na Universidade de Sonora. Nos resultados, constatou-se que as seguintes variáveis ou construtos latentes: Custos econômicos, Serviços, Imagem da universidade e Infraestrutura são adequados para modelar a variável "Escolha de carreira" por meio de um modelo de equações estruturais.

Palavras-chave: análise fatorial confirmatória, equações do modelo estrutural, escolha do 


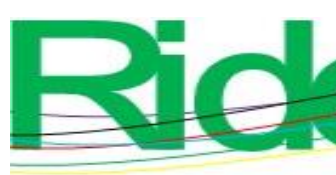

Revista Iberoamericana para la

Investigación y el Desarrollo Educativo

ISSN $2007-7467$

título universitário, instrumento de medida, validade e confiabilidade do instrumento de medida.

Fecha Recepción: Noviembre 2020

Fecha Aceptación: Mayo 2021

\section{Introducción}

La elección del programa de estudio y la universidad o instituto donde se ha de cursar este programa de estudio son factores que influyen en la deserción de los estudiantes universitarios (Nieves y Vivas, 2008; Smulders, 2018). La deserción es un indicador de eficiencia o de la calidad de las instituciones de educación superior (IES). Así, para las IES que buscan mejorar su desempeño, reducir el valor de este indicador es una tarea prioritaria. Para lograr lo anterior, es necesario identificar los factores externos que influyen en la decisión del alumno. Y no son pocos los esfuerzos de las IES, tanto del sector público como del privado, que buscan arrojar luz sobre este tema. Por ejemplo, The National Research Center for College and University Admissions (NRCCUA) realiza anualmente una encuesta a universitarios de nuevo ingreso que indaga sobre las razones por las que eligieron la universidad en la que están inscritos. En 2018, dicha encuesta, aplicada a más de 100000 estudiantes graduados de diversas escuelas secundarias de Estados Unidos, develó las siguientes siete razones clave:

1) Asequibilidad.

2) Disponibilidad de un programa deseado.

3) Reputación de la universidad.

4) Calidad académica, oportunidades de trabajo al graduarse.

5) Valor de la educación por el costo.

6) Presión social (sensación de pertenencia).

7) Proximidad a casa (Guijosa, 2018).

También hay investigaciones que han tratado este tema. López (2017) modela y describe la relación de dependencia entre la elección del programa de estudio y la universidad o instituto con los siguientes tres factores: 1) factor individual-escolar, 2) factor familiar y 3) factor institucional-contextual. Mientras que García y Moreno (2012), mediante un análisis factorial exploratorio (AFE), incluyen los factores puestos a continuación: económicos, de calidad institucional y académica, de infraestructura y administrativos. Otro estudio más es el de Montesano y Zambrano (2013), 


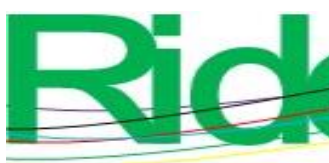

Revista Iberoamericana para la

Investigación y el Desarrollo Educativo ISSN 2007 - 7467

quienes presentan un análisis descriptivo de la relación entre la elección en cuestión con los factores económicos, de ubicación y de prestigio de la institución. Por último, Lozano (2007) y Lozano y Silva (2014) consideran la falta de motivación, la indecisión, las dificultades a la hora de autoconocerse, de buscar información y de resolver conflictos internos y externos, y validan la relación entre estos con la elección universitaria a través de un análisis factorial confirmatorio (AFC).

En este trabajo de investigación se propone validar un instrumento de medición cuya variable latente está relacionada con la elección de la carrera y la universidad por parte de los recién egresados de la educación medio superior. Elegir qué carrera o el programa de estudios y la organización en la que se ha de cursar esta carrera son dos elementos que todo aquel que desea obtener un grado universitario debe sopesar. Para la elección de una carrera, las personas se pueden apoyar en instrumentos que permiten identificar las habilidades y capacidades para el desarrollo de una profesión en particular (exámenes de ubicación) y la proyección tanto de la demanda de cada una de las opciones ofertadas como los salarios promedios a los que se puede esperar como egresado de estas.

La elección de carrera obedece a factores internos y factores externos. Los factores internos son aquellos que intrínsecamente tienen que ver con la vocación e interés del estudiante, mientras que los factores externos son todos los elementos contextuales: la familia, las amistades, la oferta educativa, duración de la carrera, seguridad, costos, los mitos profesionales y salida laboral, por mencionar algunos (Canals, 2013).

Ahora bien, validar un instrumento de medición o instrumento de medida consiste en cuantificar las relaciones entre los indicadores (que son las respuestas observadas, variables medibles o ítems) y las variables latentes, constructos o conceptos no observables (Soriano, 2014). Así, cuando la relación entre estos es significativamente fuerte, tiene sentido establecer inferencias, las cuales pueden ser planteadas y validadas mediante los modelos de ecuaciones estructurales (MES). Por lo anterior, es necesario contar con un método o procedimiento para cuantificar esta relación, además de definir los parámetros que permitan declarar significativa o no esta relación.

La exactitud (validez) y la precisión (confiabilidad) del instrumento de medición representan dos elementos que permiten cuantificar la relación entre los ítems y la variable latente, y determinar si esta es significativa, ya que con esto se garantiza que el instrumento es capaz de medir lo que tiene que medir sin variaciones en condiciones similares (Carvajal, 


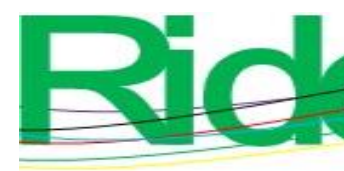

Revista Iberoamericana para la
Investigación y el Desarrollo Educativo
ISSN $2007-7467$

Centeno, Watson, Martínez y Sanz, 2011). Sin embargo, antes de llevar a cabo este análisis estadístico, es necesario, además de verificar el cumplimiento de los supuestos correspondientes a cada método estadístico, validar el contenido del instrumento de medición, esto es, cuantificar en qué medida los indicadores son relevantes y representativos de las variables latentes (Aldás y Uriel, 2017).

Diseñar un instrumento de medición para un proyecto de investigación conlleva generar un conjunto de ítems que estén relacionados con las variables latentes o constructos, de tal suerte que con estos ítems se logre recoger y reflejar todas las características propias del constructo (Clark y Watson, 2016). Para probar cualitativamente la validez del contenido del instrumento, normalmente se somete este a la opinión de un grupo de expertos en el área, y posteriormente se realiza un comparativo de las opiniones surgidas, lo cual se lleva a cabo en dos partes: primero, estos expertos lo evalúan según cuatro rubros: 1) suficiencia, 2) relevancia, 3) claridad y 4) coherencia (Escobar y Cuervo, 2008); y una vez emitidos sus juicios, se establece el grado de acuerdo entre ellos al utilizar el coeficiente Kendall o la prueba de Friedman (Abdi, 2007; Kendall y Smith, 1939).

Una vez validado el contenido, el instrumento se somete a la validación de métodos estadísticos. Los resultados de este proceso dictaminarán si es necesario eliminar algunos de los ítems. Aquí cabe traer a colación la advertencia de Aldás y Uriel (2017), quienes señalan que se debe tener cuidado con esta eliminación y no poner en riesgo la validez de contenido de inicio.

\section{Hipótesis}

Los siguientes factores externos: costos, prestigio, infraestructura y servicios que corresponden a la IES, en conjunto con el factor emotivo del alumno, influyen en los estudiantes al momento de decidir qué carrera y qué universidad elegir para su formación profesional.

\section{Objetivo general}

Desarrollar un modelo que permita relacionar los factores externos arriba mencionados con la decisión de los estudiantes de qué carrera y qué IES elegir para su formación profesional. 


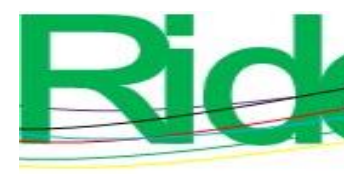
Revista Iberoamericana para la
Investigación y el Desarrollo Educativo
ISSN $2007-7467$

\section{Objetivos específicos}

1) Reducir la tasa de deserción en las instituciones de educación superior.

2) Proporcionar elementos a las IES con los cuales puedan establecer estrategias para incrementar la matrícula y elevar la calidad de los servicios educativos que ofrecen.

\section{Meta}

Validar el instrumento de medición a utilizarse en el diseño y construcción del modelo planteado en el objetivo general de este trabajo a través de los MES de estructura de covarianza.

\section{Metodología}

La metodología de esta investigación es de enfoque cuantitativo, ya que los datos recopilados son de naturaleza discreta y se utilizan para probar la hipótesis de la existencia de una relación lineal entre un conjunto de variables latentes e ítems, además de establecer el modelo que define o regula esta relación lineal, lo cual se logra mediante el uso del análisis estadístico, en particular con el uso de los MES, con el objetivo de lograr un mayor entendimiento del fenómeno bajo estudio al utilizar la información recolectada (Malhotra, 2008). El alcance de la investigación es correlacional, y tiene la finalidad de validar el instrumento de medición, y el diseño de la investigación es no experimental, ya que observa las variables bajo estudio en su contexto natural sin manipular las variables independientes; y es transeccional o transversal de tipo correlacional-causal, dado que se recolecta información en momento único, y pretende describir las relaciones entre dos o más variables, ya sea en términos correlacionales o en función de la relación causa-efecto (Hernández, Fernández y Baptista, 2014; Poblano Ojinaga, 2019).

\section{Estructura y validación del instrumento de medición}

El instrumento de medición es resultado de la revisión de la literatura. Se identificaron 13 variables medibles (13 ítems) y cinco factores o variables latentes (F1, F2, F3, F4, y F5). En la tabla 1 se muestran los ítems o variables medibles que se utilizan en este trabajo con su respectiva codificación, mientras que en la tabla 2 se presenta la asociación establecida entre los ítems y los factores considerados para este trabajo de investigación. 
Tabla 1. Ítems y su codificación

\begin{tabular}{|l|l|}
\hline ¿Qué tan importante fue para ti...? & Código \\
\hline La imagen de la universidad & IM01 \\
\hline El prestigio de la carrera que elegiste & IM02 \\
\hline Los salones y audiovisuales & IN01 \\
\hline Los laboratorios y talleres de la universidad & IN02 \\
\hline Que la universidad cuente con biblioteca & SE01 \\
\hline Que cuente con estacionamiento & SE02 \\
\hline Que cuente con cafetería & SE03 \\
\hline El costo de inscripción & EC01 \\
\hline El costo de transporte & EC02 \\
\hline El costo de libros y materiales & EC03 \\
\hline La recomendación de un conocido & EM01 \\
\hline Que haya un familiar egresado de la universidad & EM02 \\
\hline Que un amigo esté o haya estudiado en la universidad & EM03 \\
\hline
\end{tabular}

Fuente: Elaboración propia

Tabla 2. Codificación de factores e ítems

\begin{tabular}{|c|l|l|}
\hline \multirow{3}{*}{ Selección } & Factor o variable latente & Ítems \\
\hline \multirow{4}{*}{ F } & Costos económicos (F1) & EC01, EC02, EC03 \\
\cline { 2 - 3 } & Infraestructura (F2) & IN01, IN02 \\
\cline { 2 - 3 } & Imagen o prestigio de la institución (F3) & IM01, IM02 \\
\cline { 2 - 3 } & Servicios (F4) & SE01, SE02,SE03 \\
\cline { 2 - 3 } & Emotivo (F5) & EM01, EM02, EM03 \\
\hline
\end{tabular}

Fuente: Elaboración propia

Este instrumento de medición se diseñó siguiendo la teoría acorde al tema (Canals, 2013; Ruiz, 2018 y Vergara, 2017). Se trata de un cuestionario con 13 ítems, que son explicados por cinco variables latentes. La validación del contenido de este cuestionario se llevó a cabo en dos partes. Primero, se sometió a la validación de un grupo de cuatro expertos en el área, quienes lo aprobaron. Como se adelantaba líneas arriba, la validación de esta aprobación se enfocó en cuatro rubros: 1) suficiencia, 2) relevancia, 3) claridad y 4) 


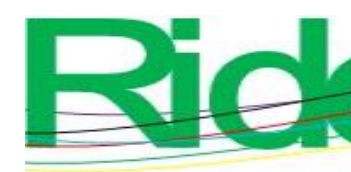

Revista Iberoamericana para la

Investigación y el Desarrollo Educativo

ISSN 2007 - 7467

coherencia (Escobar y Cuervo, 2008). Una vez emitido los juicios, se establece el grado de acuerdo entre ellos mediante el coeficiente Kendall o la prueba de Friedman para cada uno de los cuatro rubros antes mencionados (Abdi, 2007; Kendall y Smith, 1939). Para lo anterior se plantea la siguiente hipótesis para cada uno de ellos:

- $\quad \mathrm{H}_{0}$ : Todos los efectos del tratamiento son igual a cero.

Y se emplea un nivel de significancia de $5 \%$ para verificarlas con un análisis no paramétrico como alternativa al análisis bidireccional de la varianza con el empleo del paquete estadístico R.

\section{Recopilación y validación de la adecuación de la muestra}

La encuesta a utilizar para la recopilación de los datos constó de 13 preguntas, una por cada ítem considerado en el instrumento. La respuesta a cada una de estas preguntas correspondió a una escala Likert de cinco categorías: el 1 representa la categoría "Totalmente en desacuerdo", mientras que el número 5, el mayor de la escala, corresponde a "Totalmente de acuerdo". Los datos muestrales fueron tomados según la técnica de muestreo no probabilístico, por conveniencia: los encuestados son seleccionados por el hecho de que se encuentran en el lugar y el momento adecuado (Malhotra, 2008).

Este instrumento fue aplicado a 157 estudiantes que cursan la materia de Probabilidad y Estadística de la carrera de ingeniería Industrial de la Universidad de Sonora. Sin embargo, la cifra anterior fue reducida a 151 después de eliminar los valores atípicos mediante el procedimiento de la distancia de Mahalanobis con un nivel de significancia igual 0.01. Durante validación en la que se establece la idoneidad de los datos obtenidos en esta muestra para su correspondiente análisis factorial se miden, con el uso del software R, los valores de los siguientes indicadores: prueba de esfericidad de Bartlett (con la que se prueba la hipótesis nula de que la matriz varianza-covarianza de los datos de esta muestra es igual a la matriz identidad con un nivel de significancia igual a 0.05) y la medición de la adecuación muestral se verifica con la prueba de Kaiser-Meyer-Olkin (KMO), la cual mide, precisamente, la adecuación de la muestra para el análisis factorial. 


\section{Validación del instrumento de medición}

Para probar las hipótesis estadísticas que son planteadas en los MES, es necesario validar el instrumento. Y para ello se debe garantizar la identificación del modelo; la razonabilidad que incluye evaluar la bondad de ajuste y determinar si los parámetros estimados son significativos y toman valores teóricos adecuados; la validez y la confiabilidad del modelo planteado del AFC se lleva a cabo al utilizar el paquete lavaan del software $\mathrm{R}$ y con el estimador robusto insesgado de mínimos cuadrados (ULSM, por sus sigla en inglés), el cual muestra un mejor desempeño, donde el supuesto de normalidad y la identificación del modelo no se cumple (Morata, 2017).

En este trabajo se utiliza los MES, en particular el AFC, cuyo modelo se presenta en la figura 1.

Figura 1. Diagrama del modelo para el AFC

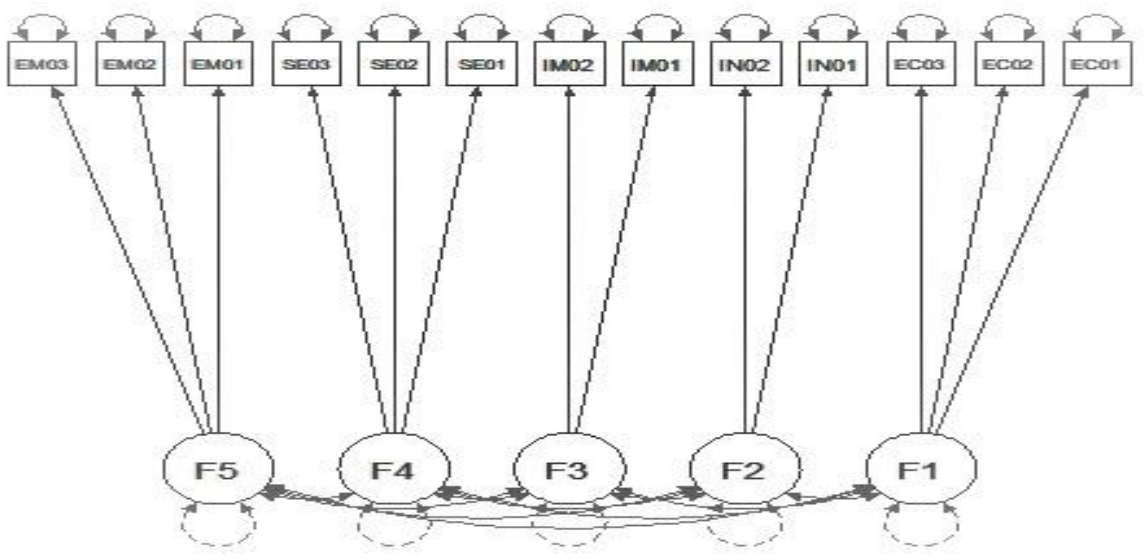

Fuente: Elaboración propia

\section{Identificación del modelo}

Identificar el modelo consiste en garantizar que el número de parámetros libres a estimar sea menor o igual al número de parámetros en la matriz varianza-covarianza del modelo, la cual tiene un número de parámetros distintos igual a $(p / 2)(p+1)$, que para este modelo con $p=13$ corresponde a 91 parámetros, mientras que las estimaciones de los parámetros del modelo requieren 36 grados de libertad, quedando libres 55 grados de libertad, lo que garantiza la identificación del modelo. 


\section{Razonabilidad del modelo}

La bondad de ajuste de este modelo propuesto se lleva a cabo comparando los valores que corresponden a los indicadores ji al cuadrado, ji al cuadrado / gl, SRMR, RMSEA, TLI, CFI, que se obtienen a partir del AFC, los cuales son comparados contra los valores críticos que se consideran para verificar esta bondad de ajuste (Aldás y Uriel, 2017).

La otra parte de la razonabilidad del modelo se considera adecuada si en los parámetros estimados del modelo no existen correlaciones superiores a la unidad, no existen cargas factoriales estandarizadas fuera del intervalo de número reales [-1,1] y ninguna de las varianzas estimadas es negativa.

\section{Validez y confiabilidad del modelo}

La validez del modelo es evaluada en tres aspectos: validez de contenido, verificada con la prueba de Friedman; validez de convergencia, que es medida con el indicador de varianza promedio extraída (AVE, por sus siglas en inglés), y la validez discriminante, que es valorada al usar el radio Heterotrait Monotrait (HTMT) y el test del intervalo de confianza. Para la valoración de la confiabilidad del modelo, se utiliza el alfa de Cronbach y la confiabilidad compuesta (CR, por sus siglas en inglés). En todos los casos se utilizan los valores críticos considerados en Aldás y Uriel (2017) y Lin (2007).

\section{Resultados}

\section{Validación del contenido del instrumento}

Los resultados emitidos por los cuatro conocedores/expertos, que evalúan el instrumento de medición en los aspectos de suficiencia, relevancia, claridad y coherencia, se presentan en la tabla 3

Tabla 3. Resultado de la prueba de Friedman

\begin{tabular}{|l|c|c|c|c|}
\hline & Suficiencia & Relevancia & Claridad & Coherencia \\
\hline S-statistic & 2.61 & 1.28 & 0.27 & 5.16 \\
\hline DF & 3 & 3 & 3 & 3 \\
\hline$P$-value & $0.457^{*}$ & $0.734^{*}$ & $0.0966^{*}$ & $0.160^{*}$ \\
\hline
\end{tabular}

*Significativo a $5 \%$

Fuente: Elaboración propia 


\section{Adecuación de la muestra}

De los 157 integrantes de la muestra, se eliminaron seis siguiendo el criterio de distancia de Mahalanobis, lo que reduce el tamaño de la muestra a 151. Los valores observados en la tabla 4 corresponden a los valores del KMO y los resultados de la prueba de la esfericidad de Bartlett aplicada a la muestra de tamaño 151.

Tabla 4. Valores de los indicadores utilizados para evaluar la adecuación la muestra

\begin{tabular}{|l|l|l|}
\hline Indicador & Valor del modelo & Valor recomendado \\
\hline Esfericidad de & $\begin{array}{l}\text { P-value }=3.28 \text {-e08, } \\
\mathrm{gl}=12\end{array}$ & P-value $<0.05$ \\
\hline Kartlett & $\mathrm{KMO}=0.73$ & $\mathrm{KMO}>0.70$ \\
\hline
\end{tabular}

Fuente: Elaboración propia

\section{Razonabilidad del modelo}

En la tabla 5 se muestran los resultados que se obtienen del AFC.

Tabla 5. Indicadores de la bondad de ajuste del modelo

\begin{tabular}{|l|l|l|l|l|l|l|}
\hline $\begin{array}{l}\text { Índice de } \\
\text { ajuste }\end{array}$ & $\begin{array}{l}\text { P-value-ji al } \\
\text { cuadrado }\end{array}$ & $\begin{array}{l}\text { Ji al } \\
\text { cuadrado/DF }\end{array}$ & CFI & TLI & RMSEA & SRMR \\
\hline Modelo & 0.000 & 1.05 & 0.997 & 0.995 & 0.019 & 0.073 \\
\hline Recomendado & 0.01 & $<3.0$ & $>0.90$ & $>0.90$ & $<0.08$ & $<0.08$ \\
\hline
\end{tabular}

Fuente: Elaboración propia

La figura 2 muestra el histograma de los valores estandarizados correspondientes a los residuos estandarizados del modelo lineal observado en relación con modelo lineal estimado, en donde se puede observar que estos residuos están distribuidos de manera simétrica, centrados en el cero con una dispersión no fuera de la distribución normal. 
Figura 2. Histograma de residuales del AFC

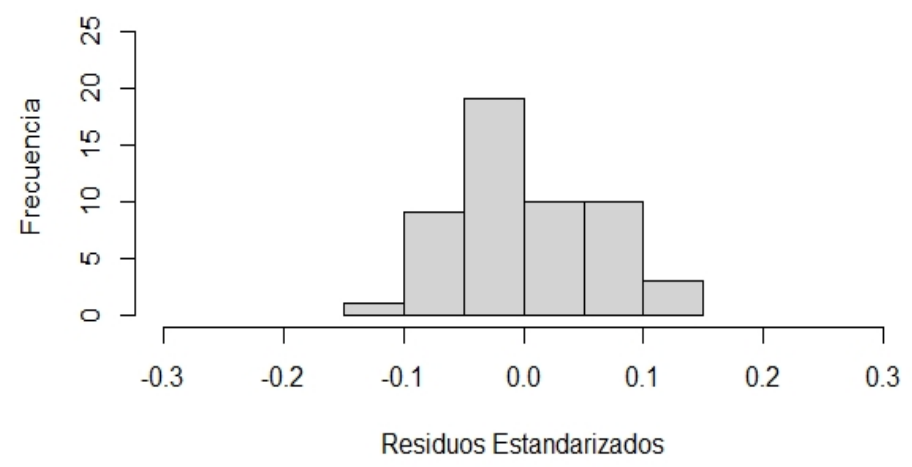

Fuente: Elaboración propia

Por otro lado, las estimaciones de los parámetros estandarizados que se obtienen en el AFC son presentados en la tabla 6.

Tabla 6. Valores estandarizados del modelo obtenido en el AFC

\begin{tabular}{|c|c|c|c|c|c|c|c|c|c|c|c|c|c|c|}
\hline$\cdot \frac{\pi}{\vec{E}}$ & \multicolumn{3}{|l|}{ F1 } & \multicolumn{3}{|l|}{$\mathrm{F} 2$} & \multicolumn{2}{|l|}{ F3 } & \multicolumn{3}{|l|}{$\mathrm{F} 4$} & \multicolumn{3}{|l|}{ F5 } \\
\hline$\stackrel{\Xi}{\Xi}$ & EC01 & $\mathrm{EC} 02$ & EC03 & IN01 & IN02 & IM01 & \multicolumn{2}{|c|}{ IM02 } & SE01 & SE02 & SE03 & EM01 & EM02 & EM03 \\
\hline 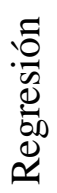 & 0.74 & 0.83 & 0.79 & 0.85 & 0.84 & 0.64 & \multicolumn{2}{|c|}{0818} & 0.726 & 0.64 & 0.77 & 0.77 & 0.78 & 0.85 \\
\hline$\cdot \frac{\sqrt[\Xi]{ే}}{>}$ & 0.45 & 0.33 & 0.32 & \multicolumn{2}{|l|}{0.27} & 0.21 & 0.43 & 0.28 & 0.27 & 0.73 & 0.45 & 0.42 & 0.56 & 0.33 \\
\hline 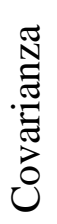 & \multicolumn{3}{|c|}{$\begin{array}{l}\mathrm{F} 2=0.42 \\
\mathrm{~F} 3=0.28 \\
\mathrm{~F} 4=0.16 \\
\mathrm{~F} 5=0.22\end{array}$} & \multicolumn{3}{|c|}{$\begin{array}{l}\mathrm{F} 3=0.56 \\
\mathrm{~F} 4=0.56 \\
\mathrm{~F} 5=0.19\end{array}$} & \multicolumn{2}{|c|}{$\begin{array}{l}\mathrm{F} 4=0.39 \\
\mathrm{~F} 5=0.39\end{array}$} & \multicolumn{3}{|c|}{$\mathrm{F} 5=0.28$} & & & \\
\hline
\end{tabular}

Fuente: Elaboración propia 


\section{Validez y confiabilidad del modelo}

En las tablas 7, 8 y 9 se muestran los valores de los indicadores para evaluar la validez y confiabilidad del modelo, los intervalos de confianza para las covarianzas de las variables latentes de este modelo y la confiabilidad del modelo a través de los valores del alfa de Cronbach y la CR.

Tabla 7. Indicadores para evaluar la validez y confiabilidad del modelo

\begin{tabular}{|c|c|c|c|c|c|c|c|c|c|}
\hline Indicador & $\begin{array}{l}\text { Alfa de } \\
\text { Cronbach }\end{array}$ & $\mathrm{CR}$ & AVE & \multicolumn{6}{|c|}{ Criterio de Fornell y Larcker } \\
\hline \multirow{6}{*}{$\begin{array}{l}\text { Valor } \\
\text { observado en } \\
\text { el modelo }\end{array}$} & $\mathrm{F} 1=0.83$ & $\mathrm{~F} 1=0.83$ & $\mathrm{~F} 1=0.61$ & & F1 & $\mathrm{F} 2$ & F3 & F4 & $\mathrm{F} 2$ \\
\hline & $\mathrm{F} 2=0.82$ & $\mathrm{~F} 2=0.82$ & $\mathrm{~F} 2=0.71$ & F1 & 0.620 & & & & \\
\hline & $\mathrm{F} 3=0.68$ & $\mathrm{~F} 3=0.70$ & $\mathrm{~F} 3=0.54$ & F2 & 0.193 & 0.705 & & & \\
\hline & $\mathrm{F} 4=0.73$ & $\mathrm{~F} 4=0.76$ & $\mathrm{~F} 4=0.52$ & F3 & 0.077 & 0.308 & 0.536 & & \\
\hline & \multirow{2}{*}{$\mathrm{F} 5=0.84$} & \multirow{2}{*}{$\mathrm{F} 5=0.84$} & \multirow{2}{*}{$\mathrm{F} 5=0.64$} & $\mathrm{~F} 4$ & 0.025 & 0.312 & 0.156 & 0.519 & \\
\hline & & & & F5 & 0.047 & 0.059 & 0.155 & 0.076 & 0.639 \\
\hline $\begin{array}{l}\text { Valor } \\
\text { recomendado }\end{array}$ & $>0.70$ & $>0.70$ & $>0.50$ & \multicolumn{6}{|c|}{$\begin{array}{l}\text { Valores de la diagonal principal mayoro igual } \\
\text { que cualquier valor del renglón o columna } \\
\text { correspondiente. }\end{array}$} \\
\hline
\end{tabular}

Fuente: Elaboración propia 
Tabla 8. Intervalos de confianza de $95 \%$ para la covarianza

\begin{tabular}{|c|c|c|}
\hline Covarianza & Límite inferior & Límite superior \\
\hline F1 F2 & 0.22 & 0.61 \\
\hline F1 F3 & 0.05 & 0.50 \\
\hline F1 F4 & 0.08 & 0.40 \\
\hline F1 F5 & 0.01 & 0.42 \\
\hline F2 F3 & 0.36 & 0.74 \\
\hline F2 F4 & 0.36 & 0.73 \\
\hline F2 F5 & -0.02 & 0.40 \\
\hline F3 F4 & 0.17 & 0.61 \\
\hline F3 F5 & 0.18 & 0.60 \\
\hline F4 F5 & 0.03 & 0.51 \\
\hline Valor recomendado: límite superior <1.0 \\
\hline \multicolumn{2}{|c|}{ Fuente: Elaboracion propia } \\
\hline
\end{tabular}

Fuente: Elaboración propia

Tabla 9. Valores del HTMT para el modelo AFC del SEM

\begin{tabular}{|l|l|l|l|l|l|}
\hline & F1 & F2 & F3 & F4 & F5 \\
\hline F1 & 1.000 & & & & \\
\hline F2 & 0.411 & 1.000 & & & \\
\hline F3 & 0.278 & 0.559 & 1.000 & & \\
\hline F4 & 0.240 & 0.637 & 0.468 & 1.000 & \\
\hline F5 & 0.240 & 0.205 & 0.392 & 0.281 & 1.000 \\
\hline Valor recomendado: < 0.90 para los valores fuera de la \\
diagonal principal.
\end{tabular}

Fuente: Elaboración propia

\section{Discusión de resultados}

De acuerdo con los resultados de la tabla 3 , el $p$-value para cada rubro es mayor que 0.05, por lo que no hay evidencia suficiente para rechazar la $\mathrm{H}_{0}$ para cada uno de estos rubros. Por lo tanto, se puede concluir que existe concordancia entre los juicios que emiten los expertos y así declarar válido el contenido del instrumento de medición. 


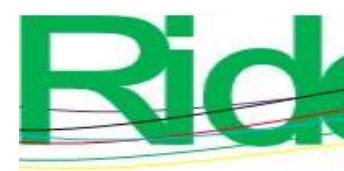

Revista Iberoamericana para la
Investigación y el Desarrollo Educativo
ISSN $2007-7467$

En la tabla 4, por su parte, se muestran los valores de los indicadores de la prueba de esfericidad de Bartlett y el valor del índice KMO. A partir de estos no es posible rechazar la hipótesis nula de que la matriz varianza-covarianza de los datos de esta muestra es igual a la matriz identidad con un nivel de significancia igual a 0.05 , de lo que se deriva que la matriz covarianza de estos datos es diferente de la matriz identidad; mientras que el valor del índice KMO igual a 0.73 cae en la categoría de meritorio referida por Aldás y Uriel (2017). De acuerdo con los resultados anteriores, se concluye que esta muestra es adecuada para el análisis factorial.

Los valores observados en la tabla y el comportamiento de los residuales observados en la figura 2 permiten asumir que el ajuste del modelo es adecuado, al verificar el cumplimiento de la bondad de ajuste del modelo estimado.

En la tabla 6 se observa que no existen correlaciones superiores a la unidad ni existen cargas factoriales estandarizadas fuera del intervalo [-1,1], y ninguna de las varianzas estimadas es negativa. Estos resultados, en conjunto con la bondad del ajuste del modelo, llevan a concluir que la razonabilidad del modelo es adecuada.

Los valores del indicador CR observados en la tabla 7 sobrepasan o igualan el valor recomendado, mientras que los valores del indicador alfa de Cronbach sobrepasan los valores recomendados, con excepción de la variable latente F3, cuyo valor está próximo al valor recomendado. En esta situación se considera que el instrumento de medición satisface el criterio de confiabilidad, dado que la CR es un criterio más robusto en comparación del alfa de Cronbach (Fornell y Larcker, 1981).

Los valores de la AVE mostrados en la tabla 7 sobrepasan los valores recomendados, lo que lleva a establecer que este instrumento de medición cumple con el criterio de la validez convergente.

Los valores de los tres criterios para evaluar la validez divergente en el instrumento de medición, a saber, Fornell y Larcker, intervalos de confianza para la covarianza y HTMT, que se muestran en la tabla 7, 8 y 9, respectivamente, superan en los tres casos los valores recomendados, por lo que se establece que el instrumento tiene validez divergente.

La validación de este instrumento siguió el procedimiento establecido en los MES de estructura de covarianza. El análisis preliminar de la validez y la confiabilidad llevado a cabo en el AFC sugiere la necesidad de eliminar una variable latente y un ítem de otra de estas variables con el objetivo de poder garantizar la validez convergente del instrumento, y 


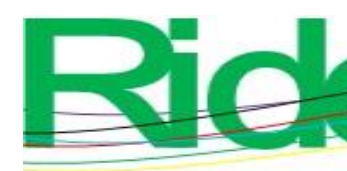

Revista Iberoamericana para la

Investigación y el Desarrollo Educativo

ISSN 2007 - 7467

una vez realizada esta eliminación se cumple con las características tanto de validez convergente y discriminante como de confiabilidad.

En este caso, como consecuencia del análisis estadístico correspondiente, la ubicación de la universidad es la variable latente que se elimina, ya que el valor calculado del AVE no cumple con el valor mínimo requerido, lo cual, desde el punto de vista estadístico, indica que las cargas factoriales de los ítems considerados no presentan la correlación necesaria para explicar la variabilidad observada, y esta última debe atribuirse al error de los ítems, lo que lleva a interpretar que esta variable latente no tiene ningún peso específico para este grupo de alumnos sobre aquello que los llevó a escoger la carrera y la universidad en la que se encuentran.

\section{Conclusiones}

A partir de los resultados presentados en la sección anterior se concluye que el instrumento de medición tiene validez de contenido, que la muestra es adecuada y que el modelo del AFC es razonable, es confiable y válido. En suma, se concluye que el IM es válido y confiable, esto es, resulta adecuado para medir los criterios que influyen en los alumnos para elegir su carrera universitaria. Y dado su nivel de confiabilidad y validez, los datos obtenidos a través de este instrumento son susceptibles de someterse a las pruebas de hipótesis de los MES correspondientes.

Lo anterior implica que las variables latentes Costos (F1), Infraestructura (F2); Imagen o prestigio de la institución (F3), Servicios (F4) y Emotivos (F5) satisfacen la condición necesaria para que estas sean consideradas como factores que influyen en la decisión de los estudiantes al momento de escoger qué carrera y qué IES elegir para su formación profesional.

Con los resultados de la evaluación de este instrumento, y con la validación de las hipótesis planteadas sobre las relaciones que guardan esta variables latentes, las IES públicas o privadas pueden plantear estrategias para minimizar el índice de deserción con programas tendientes a reducir el peso o carga que tienen los factores externos que hacen que el alumno seleccione la IES, el programa o carrera universitaria que no corresponda a su vocación o interés intrínseco y, por ello, decida abandonar este programa, además de permitirles generar programas de mercadotecnia con el objetivo de incrementar la matrícula. 


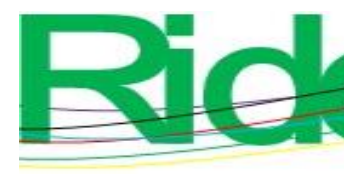

Revista Iberoamericana para la

Investigación y el Desarrollo Educativo ISSN 2007-7467

\section{Futuras Líneas de investigación}

- $\quad$ Plantear y validar las hipótesis sobre las formas en que se relacionan las cinco variables latentes validadas en este instrumento de medición a través de los MES de estructura de covarianza. Y para esto se sugiere plantear el SEM de segundo orden.

- $\quad$ Evaluar este instrumento de medición en diferentes IES para verificar su utilidad como herramienta para validar las hipótesis que se plantean sobre la relación y el impacto que tienen las seis variables latentes (factores externos), Costos, Ubicación, Infraestructura, Imagen o prestigio, Servicios y Emotivos, sobre la decisión que debe tomar el alumno al momento de decidir qué carrera seleccionar y en qué IES cursarla.

- Elaborar un modelo que resulte útil para cuantificar el impacto que tienen estos seis factores externos sobre la decisión que debe tomar el alumno al momento de decidir qué carrera seleccionar y en qué IES cursarla, y a su vez, medir o cuantificar la relación que guarda esta decisión en relación con la variable deserción escolar en las IES.

Para utilizar el MES de estructura de covarianza se requiere verificar que los datos correspondientes a los ítems sigan una distribución normal multivariante, que no presenten colinealidad y que el tamaño de la muestra sea mayor que el número de variables latentes. De estas tres condiciones, en la práctica, y sobre todo cuando en los instrumentos se utiliza la escala Likert, la condición de distribución normal multivariada es la más difícil de cumplir. Sin embargo, existen métodos de estimación en los que esta condición es solventada tomando un tamaño de muestra adecuado. Además, se puede disponer de métodos no paramétricos (métodos robustos y bootstrap) en los cuales esta normalidad no es requerida. La colinealidad puede resolverse a través del diseño apropiado de las preguntas en el instrumento. Pero resulta imposible realizar el análisis bajo el enfoque de los ES de estructura de covarianza cuando la cantidad de variables latentes es mayor que el tamaño de la muestra.

El modelo de ecuaciones estructurales con mínimos cuadrados parciales es un método alternativo para validar los instrumentos de medición y validar las hipótesis que se planteen con respecto a la relación que guardan las variables latentes. Este modelo no requiere del cumplimiento de los tres supuestos establecidos en el MES de estructura de covarianza, además de que con aproximadamente $20 \%$ del tamaño de la muestra que requiere el MES de estructura de covarianza es suficiente. 


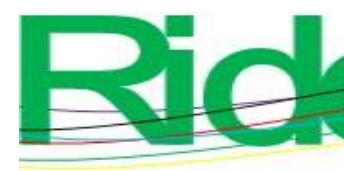

Revista Iberoamericana para la
Investigación y el Desarrollo Educativo
ISSN $2007-7467$

Dado lo anterior se plantea realizar un nuevo trabajo de investigación que emprenda la comparación entre los resultados que se obtienen al evaluar el instrumento con el MES de estructura de covarianza con los obtenidos por el de mínimos cuadrados parciales.

\section{Referencias}

Abdi, H. (2007). The Kendall Rank Correlation Coefficient. In Salkind, N. (ed.), Encyclopedia of Measurement and Statistics. Thousand Oaks, United States: Sage.

Aldás, M. J. y Uriel, J. E. (2017). Análisis multivariante aplicado con R (2. ${ }^{a}$ ed.). Madrid, España: Ediciones Paraninfo.

Canals, C. (2013). Factores que inciden en la elección de escuela. (Tesis doctoral). Universidad de Chile, Santiago. Recuperado de http://repositorio.uchile.cl/handle/2250/115554.

Carvajal, A., Centeno, C., Watson, R., Martínez, M. y Sanz, Á. (2011). ¿Cómo validar un instrumento de medida de la salud? Anales del Sistema Sanitario de Navarra, 34(1), 63-72.

Clark, L. A. and Watson, D. (2016). Constructing validity: Basic issues in objective scale development. In Kazdin, A. E. (ed.), Methodological Issues and Strategies in Clinical Research (pp. 187-203). United States: American Psychological Association. Retrieved from https://doi.org/10.1037/14805-012.

Escobar, J. y Cuervo, Á. (2008). Validez de contenido y juicio de expertos: una aproximación a su utilización. Avances en Medición, 6(1), 27-36.

Fornell, C. and Larcker, D. F. (1981). Evaluating structural equation models with unobservable variables and measurement error. Journal of Marketing Research, $18(1), 39-50$.

García, J. y Moreno, C. (2012). Factores considerados al seleccionar una universidad: caso Ciudad Juárez. Revista Mexicana de Investigación Educativa, 17(52), 287-305.

Guijosa, C. (16 de febrero de 2018). ¿Qué motiva a los estudiantes a elegir su universidad? Observatorio de Innovación Educativa. Recuperado de https://observatorio.tec.mx/edu-news/que-motiva-a-estudiantes-a-elegiruniversidad.

Hernández, R., Fernández, C. y Baptista, P. (2014). Metodología de la investigación. Ciudad de México, México: McGraw-Hill. 


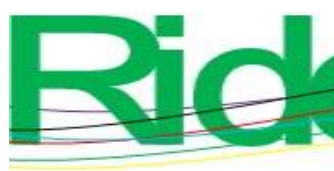

Revista Iberoamericana para la

Investigación y el Desarrollo Educativo

ISSN $2007-7467$

Kendall, M. G. and Smith, B. B. (1939). The problem of $\mathrm{m}$ rankings. The Annals of Mathematical Statistics, 10(3), 275-287.

Lin, H. (2007). Predicting consumer intentions to shop online: An empirical test of competing theories. Electronic Commerce Research and Applications, 6(4), 433-442.

López, M. (2017). Elección de universidad: área de estudio y carrera: estudiantes de bachilleratos públicos y privados de Hermosillo. (Tesis de maestría). Universidad de Sonora, Hermosillo.

Lozano, R. y Silva, D. (2014). Criterios de los estudiantes que determinan la elección de una carrera universitaria en el área de negocios. Un estudio descriptivo: caso Chile. Ponencia presentada en la XIX Conferencia Internacional de Contaduría, Administración e Informática. Ciudad de México, del 8 al 10 de octubre de 2014.

Lozano, S. (2007). Validación de un modelo de medida de las dificultades en los procesos de toma de decisiones sobre la carrera profesional. Revista de Educación, (343), 325351.

Malhotra, N. (2008). Investigación de mercados (5. ${ }^{\mathrm{a}}$ ed.). Ciudad de México, México: Pearson Educación.

Montesano, J. C. y Zambrano, E. (2013). Factores que influyen en la elección de una carrera universitaria en la Universidad Católica Andrés Bello. (Trabajo de grado). Universidad Andrés Bello, Caracas.

Morata, M. (2017). Métodos de estimación y sus implicaciones para la validación de constructo mediante análisis factorial confirmatorio de escalas tipo Likert: un estudio de simulación. (Tesis doctoral). Universidad Nacional de Educación, España.

Nieves, M. E. y Vivas, L. J. (2008). Factores de deserción estudiantil en el programa de Contaduría Pública de la Universidad Libre sede principal en el período comprendido entre el semestre I de 2004 al II de 2007. (Trabajo de grado). Universidad Libre, Bogotá.

Poblano Ojinaga, E. R. (2019). Modelo Estructural de los Factores Críticos de Éxito de la Inteligencia Competitiva basado en la Administración del Conocimiento. Departamento de Ingeniería Industrial y Manufactura.

Ruiz, M. A. (2018). Factores que influyen en la deserción de los alumnos del primer ciclo de educación a distancia en la Escuela de Administración de la Universidad Señor de 


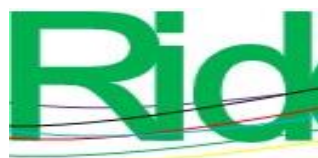

Sipán: períodos académicos 2011-1 al 2013-1: lineamientos para disminuir la deserción. Educación, 27(52), 160-173.

Smulders, A. M. (2018). Factores que influyen en la deserción de los estudiantes universitarios. Academo: Revista de Investigación en Ciencias Sociales y Humanidades, 5(2), 129-130.

Soriano, A. (2014). Diseño y validación de instrumentos de medición. Diá-logos, (14), 1940.

Vergara, J., Boj del Val, E., Barriga, O. A. y Díaz, C. (2017). Factores explicativos de la deserción de estudiantes de pedagogía. Revista Complutense de Educación, 28(2), 609-630. 


\begin{tabular}{|c|c|}
\hline Rol de Contribución & Autor(es) \\
\hline Conceptualización & $\begin{array}{l}\text { Rafael García Martínez (principal), Eduardo Rafael Poblano- } \\
\text { Ojinaga (igual), Rigoberto Reyes Valenzuela (que apoya). }\end{array}$ \\
\hline Metodología & $\begin{array}{l}\text { Rafael García Martínez (principal), Eduardo Rafael Poblano- } \\
\text { Ojinaga (igual). }\end{array}$ \\
\hline Software & $\begin{array}{l}\text { Rafael García Martínez (principal), Guillermo Cuamea Cruz } \\
\text { (igual). }\end{array}$ \\
\hline Validación & $\begin{array}{l}\text { Rafael García Martínez (principal), Eduardo Rafael Poblano- } \\
\text { Ojinaga (igual), Guillermo Cuamea Cruz (que apoya). }\end{array}$ \\
\hline Análisis Formal & $\begin{array}{l}\text { Rafael García Martínez (principal), Guillermo Cuamea Cruz } \\
\text { (que apoya) }\end{array}$ \\
\hline Investigación & $\begin{array}{l}\text { Rafael García Martínez (principal), Eduardo Rafael Poblano- } \\
\text { Ojinaga (igual), Rigoberto Reyes Valenzuela (que apoya), } \\
\text { Rubén Juárez Rodríguez (que apoya). }\end{array}$ \\
\hline Recursos & $\begin{array}{l}\text { Rigoberto Reyes Valenzuela (que apoya), Rubén Juárez } \\
\text { Rodríguez (que apoya). }\end{array}$ \\
\hline Curación de datos & $\begin{array}{l}\text { Rafael García Martínez (principal), Eduardo Rafael Poblano- } \\
\text { Ojinaga (igual). }\end{array}$ \\
\hline $\begin{array}{l}\text { Escritura - Preparación del } \\
\text { borrador original }\end{array}$ & $\begin{array}{l}\text { Eduardo Rafael Poblano-Ojinaga (principal), Rigoberto Reyes } \\
\text { Valenzuela (que apoya), Rubén Juárez Rodríguez (que apoya). }\end{array}$ \\
\hline $\begin{array}{l}\text { Escritura - Revisión y } \\
\text { edición }\end{array}$ & $\begin{array}{l}\text { Rafael García Martínez (principal), Eduardo Rafael Poblano- } \\
\text { Ojinaga (igual), Guillermo Cuamea Cruz (que apoya). }\end{array}$ \\
\hline Visualización & $\begin{array}{l}\text { Rafael García Martínez (principal), Eduardo Rafael Poblano- } \\
\text { Ojinaga (igual), Guillermo Cuamea Cruz (que apoya). }\end{array}$ \\
\hline Supervisión & $\begin{array}{l}\text { Guillermo Cuamea Cruz (principal), Rigoberto Reyes } \\
\text { Valenzuela (igual), Rubén Juárez Rodríguez (que apoya) }\end{array}$ \\
\hline Administración de Proyectos & $\begin{array}{l}\text { Rafael García Martínez (principal), Eduardo Rafael Poblano- } \\
\text { Ojinaga (igual). }\end{array}$ \\
\hline Adquisición de fondos & $\begin{array}{l}\text { Rigoberto Reyes Valenzuela (principal), Rubén Juárez } \\
\text { Rodríguez (igual) }\end{array}$ \\
\hline
\end{tabular}

